\title{
Optimized Perceptual Quality, Robust and Highly Secured Randomly Scrambled Image Watermarking using 3-Level DWT Domain
}

\author{
Shveti Sejpal \\ Research Scholar, \\ Saurashtra University, \\ Rajkot, Gujarat, India.
}

\author{
Nikesh Shah, PhD \\ Professor, \\ Department of Physics, \\ Saurashtra University, Rajkot, Gujarat, India.
}

\begin{abstract}
In networks the content distribution through genuine channels needs the use of digital watermarks. The digital watermark is to make the content self-identifying i.e. to place the information within the content itself. Digital images are the significant vector for network communication. As 2D-image signals are non-stationary signal wavelet transform is more suitable to analyze this signal. This paper presents a blind digital image watermark embedding technique based on random Arnold scrambling i.e. highly secure and its comparative performance analysis based on 3-level Discrete Wavelet Transform (DWT) domain for hiding the watermark information in cover image. It is seen that using 3-level DWT, watermarked image quality is improved compared to 1-level DWT and 2-level DWT. This technique also improves the security of image more effectively. This approach uses two frequency sub-bands $\mathrm{HL}$ and $\mathrm{HH}$ of DWT to embed watermark image to confirm the human perception characteristics. Here to achieve better robustness, better perceptual quality and efficient security watermark image is first scrambled randomly using Arnold transform and the watermark bits are distributed to the wavelet coefficients of the image edges in HLHHHH \& HHHHLH sub-bands. Here for DWT decomposition 'Haar' wavelet is used. Here gain factor $\mathrm{K}=0.55$ is selected for testing the performance under several attacks. In 3-level DWT domain good perceptual quality is achieved with PSNR value 41.5848, SSIM value 0.953 and NC value 0.663. The Proposed method is also found more robust by experimenting with different attacks. The experimental results proved that proposed technique i.e. 3-level DWT is faster with compared to 2-level DWT, 1-level DWT.
\end{abstract}

\section{General Terms}

Information hiding, Digital Watermarking, Perceptual quality, Robustness, Security.

\section{Keywords}

Digital watermarking, 3-level Discrete Wavelet Transform, Arnold Transform, Information Hiding in Image, Robustness.

\section{INTRODUCTION}

In the digital age, we all are wired in, where the digital data like entertainment media, online transactions, surveillance images and video, medical images, consumer images, etc. can be copied with just a keystroke. Very easily the images can be modified using many photographic software. The authenticity of content and the Copy right protection, for authors and owners and are the crucial factors to be solved. The actual content should be marked with the payload by the content right holders. Digital watermarking is the technique to mark the content with some copyright data permanently. It is the branch of information hiding. The content can be image, audio or video. Watermarking techniques can be categorize as blind watermarking and non-blind watermarking. In non-blind watermarking technique original image is required to extract the watermark while in blind watering scheme, no need of original image for extracting the watermark. There are basically two techniques to embed the digital watermark signal in the content, spatial domain and transform domain. While embedding the watermark in the content the perceptual quality of the content should not be degraded and also ensure that the copyright data should be impossible or difficult to remove. [1] Compared to spatial domain the robustness and higher payload capacity can be achieved through transform domain. Followings are the applications where digital image watermarking based security solutions can play a key role: copy and playback control, forensic tracking applications to collect evidence for criminal actions, Fingerprinting, MMS in Mobile, e-voting Systems, insurance companies, banking services for securing image database and their transmission, medical images, in content tracking environment. Within existing distributed system, $\mathrm{P} 2 \mathrm{P}$ systems and online communities can regulate copyrighted from no copyrighted content files through digital watermarking. Digital watermarking technique, offers noticeable and inspiring advantages for anti-piracy efforts and rights management, they are enhancing consumers' online experience and encouraging innovation in the digital marketplace. Specifically in this paper first the image is scrambled using Arnold transform with random strategies, then the watermark message has been embedded in HLHHHH and HHHHLH band through 3-level Discrete Wavelet Transform. Thus Arnold transform is combined with 3-level DWT. This paper is organized in 6 section: Section-II gives survey of work related to DWT and scrambling technique, section-III gives theoretical background, in section-IV Proposed Technique is presented. Experimentation and results are particularized in section-V while conclusion is drawn in section-VI.

\section{RELATED WORK}

Earlier as watermark embedding technique Spatial Domain was used. It has the advantage of easy implementation and low computational complexity but it is fragile to some common attacks. Ramani K. et al. [2] proposed the LSB based watermarking technique in spatial domain, here to determine pixels to be use for embedding pseudo random generator is used, based on the given seed or key is the straightforward method, but this is not completely secure algorithm. In order to overcome these shortcomings, M. Raval, P. Rege [3] has proposed the digital watermarking technique based on Discrete Wavelet Transforms. The high and low frequencies 
sub-bands are used to construct the DWT multi watermarking scheme. The combination of the two sub-bands for embedding the watermark provides a system with good robustness in a large scale. M. Chandra has used middle frequency band to add watermark image bits in the cover image [4]. Here our proposed method is more robust and secure compared to this method. Nikita Kashyap [5] proposed 3-level DWT for robust digital image watermarking technique for embedding the invisible watermark in to the salient features of image. This algorithm has used alpha blending technique for embedding the watermark in LL sub-band. The experimental result shows that the quality of watermark image and the recovered image is improved. C. Pradhan et. al. [6] has proposed effective and secure digital watermarking technique using Arnold Transform and cross Chaos map in DCT domain. In "A DWT ordering Scheme for Hiding Data in Images using Pixel Value Difference" [7] the data is hidden in cover image based on a prioritized ordering of the content in the host image. Here the host image is decomposed by using two level DWT. These sub-bands were analyzed and transformed using pixel value difference. The Most Significant Bits (MSB) of each pixel of the watermark image are hidden in the best wavelet sub-band, the HHLL region and the Least Significant Bits (LSB) are spared in HLHH region. The remaining bits are hidden in the remaining 6 sub-bands of the cover image. Using this technique the cover image can accommodate a watermark image with appreciable visual quality. Nag, A. et. al. has proposed in image encryption using affine transformation and XOR operation [8], a location transform based encryption system. They have used affine transform technique with four 8-bit keys to redistribute the pixel values to the different location. Then divided the transformed image into $2 \times 2$ pixel blocks and each block encrypted using XOR operation by four 64 bit key size. Resulted in significantly reduced correlation between pixel values. In Arnold scrambling method because of fixed form matrix, the image is easily attacked. In [9] Fermat Number transformation matrix has been combined with FFT presents secondary scrambling method that is based on location space and color space. M. Mohammed [10] proposed an innovative scheme in which the Arnold scrambled watermark is embed in high frequency sub-band of DWT on rescaled version of original image. A. Ravindran et. al. [11] has proposed Arnold transform of secret image and then DWT is applied on this transformed secret image. This secret image is embedded into the cover image. To recover watermark image IDWT and inverse Arnold transform is applied to the watermarked image.

\section{THEORETICAL BACKGROUND 3.1 Discrete Wavelet Transform}

The Discrete Wavelet Transform (DWT) is the most useful and advanced transform domain watermarking technique. It is a hierarchical transform. DWT has the capabilities to study or analyze a signal at different levels i.e. multi resolution analysis. [12] Although for image analysis Discrete Cosine
Transform (DCT) is a better transformation, the better alternate to DCT is the Discrete Wavelet Transform (DWT)

because in DWT the image is not required to divide into nonoverlapping 2-D blocks and it allows localization in both time frequency and spatial frequency. In DWT, transformation is applied to whole image, not on block by block basis. It uses integral scaling with a high compression ratio. DWT has higher flexibility in choosing the type of wavelet function like Daubechies, Coiflet, Harr, Marr, Morlet, etc. It can scale up, that smoothly produces the changes and acquires low frequency details. Wavelets can also scale down to produce rapid changes and acquire high frequency details. The DWT splits the signal into high and low frequency bands. The high frequency part contains the edge components information and the low frequency part is split again into high and low frequency parts.

\subsection{Selection of Wavelet Coefficients and Gain Factor}

As the human eye is less sensitive to changes in edges and embedding the watermark in to the cover image is same as the addition of noise to the cover image, usually for watermarking high frequency components are used. Modification in high frequency coefficient is not detected by human necked eyes. After the first level of decomposition it separates an image into a detail component band i.e. lower resolution approximation image (LL) bands, horizontal (HL), vertical $(\mathrm{LH})$ and diagonal $(\mathrm{HH})$. Embedding watermarks in these regions increase the robustness of our watermark, with not much effect on image quality. This procedure can then be repeated to obtain multiple scale of wavelet decomposition until some final scale " $\mathrm{N}$ " is reached. As shown in figure 1, after $\mathrm{N}$ scale decomposition it has $3 \mathrm{~N}+1$ sub-bands consisting of the multi-resolution sub-bands (LLN) and (LHm), (HLm) and $(\mathrm{HHm})$ where " $\mathrm{m}$ " ranges from 1 until " $\mathrm{N}$ ". [13] The numbers of tests are carried out for selection of level of decomposition of the image and sub-band to be chosen for embedding the watermark in the cover image by considering the performance of the different sub-band at different levels. The cover image was decomposed with 1-level DWT using 'HAAR' wavelet and the watermark is embedded with various gain factor $\mathrm{k}$ and using PN Sequences. The PSNR is calculated between cover image and watermarked image. Normalized correlation is calculated between original Watermark image and the extracted watermark image. From the figure 2 and figure 3 it is clear that PSNR and NC values are better in HL \& HH sub-band combination. The 1-level DWT, 2-level DWT and 3-level DWT decomposition using 'HAAR' wavelet and gain factor $\mathrm{k}=0.15$ to $\mathrm{K}=10$ is applied. In 1-level DWT decomposition watermark is embedded in HL \& $\mathrm{HH}$ sub-band of cover image, in 2-level decomposition watermark is embedded in HHHH\& HLHH band and in $3-$ level decomposition watermark is embedded in HHHHLH and HLHHHH sub-band. 


\begin{tabular}{|l|l|l|l|l|}
\hline 2D - Image & & LL & LH \\
\hline & & \\
\hline
\end{tabular}

Figure 1: Fundamental process of discrete wavelet transform on image

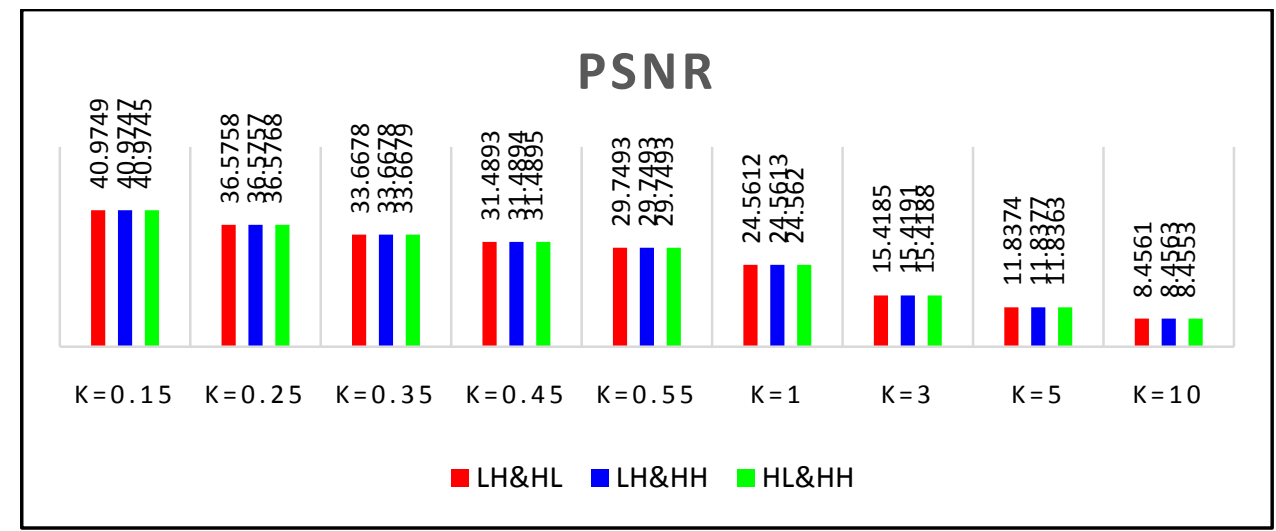

Figure 2: PSNR vs sub-band for various gain factors on watermarked image

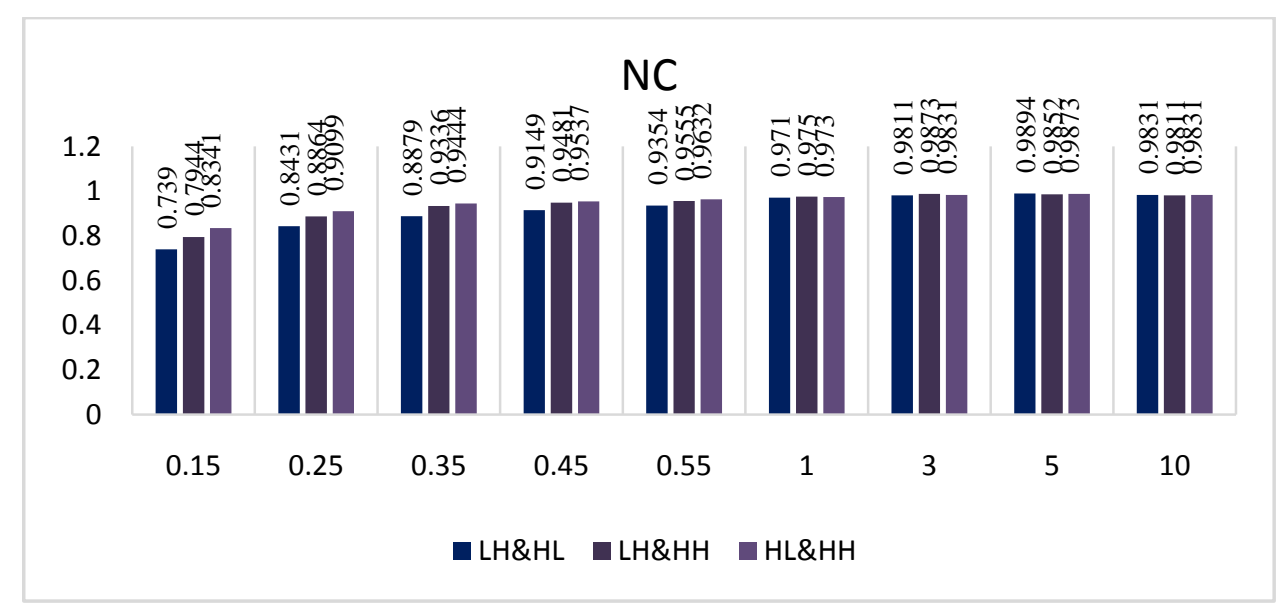

Figure 3: NC vs sub-band for various gain factors on watermarked image

The bigger PSNR value denotes better perceptual quality of watermarked image. The figure 4 and 5 shows that in case of 3-level DWT decomposition the value of the PSNR \& SSIM is higher for different values of $\mathrm{K}$. Hence the watermark bit is embedded in best sub-band HLHHHH \& HHHHLH in the proposed system. The larger value of $\mathrm{K}$ degrades the perceptual transparency of the cover image but small value of $\mathrm{k}$ degrades the robustness of the image, thus $\mathrm{K}$ must be in the reasonable range. $\mathrm{K}=0.55$ is selected for the proposed technique. 


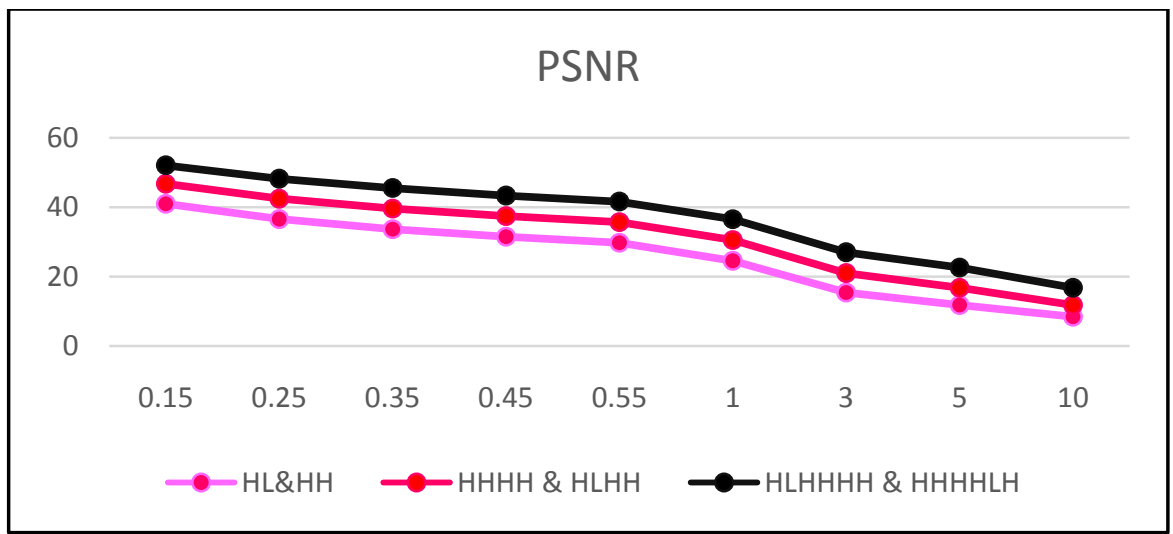

Figure 4: PSNR vs sub-band in 1-level decomposition, 2-level decomposition and 3-level decomposition for various gain factors on watermarked image

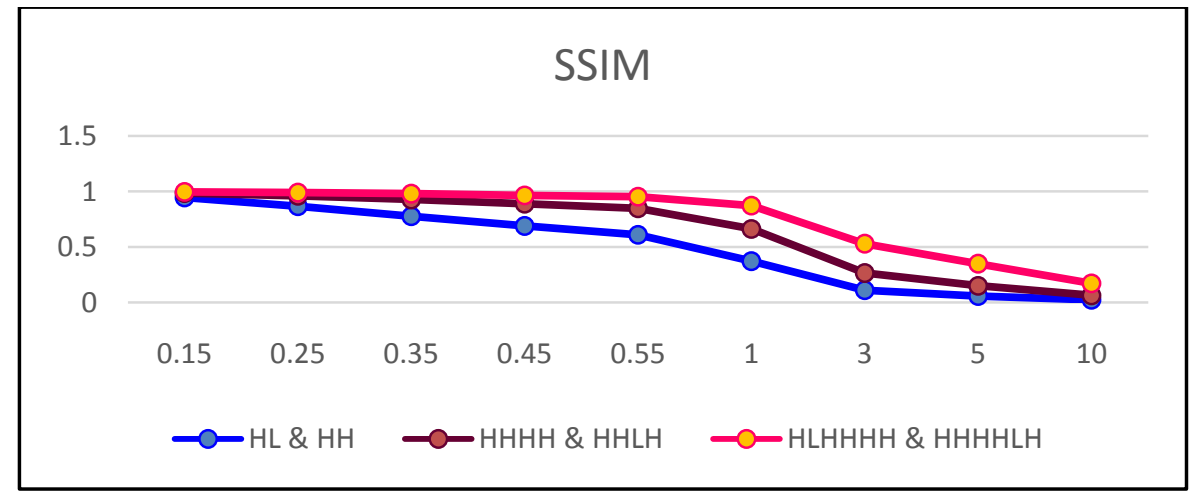

Figure 5: SSIM vs sub-band in 1-level decomposition, 2-level decomposition and 3-level decomposition for various gain factors on watermarked image

\subsection{Arnold Transfer}

As Data hiding technology brought many new algorithms, it is a revolution in the warfare of network information. The core idea is to use public network for trusted data transmission. The image after scrambling encryption algorithms is disordered, so attacker cannot decipher it. To improve the security of image, Image scrambling is used as an encryption method. Due to the periodicity and simplicity of the Arnold transform, the image can easily be recovered after the transformation. A digital image is a $2 \mathrm{D}$ function $\mathrm{f}(\mathrm{x}, \mathrm{y})$ (2-D image), the amplitude of $f$ at any pair of $(x, y)$ is grey level of the image at that point e.g, a grey level image can be represented as: $f i j=f(x i, y j)$ where $X, Y \in\{0,1,2,3, \ldots \ldots \ldots, N-$ 1) where $\mathrm{N}$ is the order of the image. Arnold Transform is also known as cat face transform is a mapping function and is only suitable for $\mathrm{N} \times \mathrm{N}$ digital images specified in many literature. The transformation of point $(\mathrm{x}, \mathrm{y})$ in the unit square changes to another point $\left(X^{\prime}, Y^{\prime}\right)$ by the following equation:

$\left[\begin{array}{l}X^{\prime} \\ Y^{\prime}\end{array}\right]=\left[\begin{array}{ll}1 & 1 \\ 1 & 2\end{array}\right]\left[\begin{array}{l}X \\ Y\end{array}\right](\bmod N)$ Equation (1)

i.e.

$\mathrm{X}^{\prime}=\mathrm{X}+\mathrm{Y} \bmod \mathrm{N}$

$Y^{\prime}=X+2 Y \bmod N$

Repeating the Transform several time (n) it changes the position of pixels and scrambled image is obtained. The period of Arnold scrambling is related to the size of the image, but not directly proportional.

Iterative process is as following:
$\underset{\left(X^{\prime}, Y^{\prime}\right)}{n+1}=\boldsymbol{A} \underset{(X, Y)}{n}(\bmod n)$ Equation (2)

A is the Arnold transform matrix and $\mathrm{n}$ is the time of iterations, $\mathrm{n}=0,1,2 \ldots$ Iteration number (n) is used as the encryption key, it is used as a secret key for extracting the secret image. The decryption of image depends on iteration number (n). Scrambling recovery of Arnold scrambled image has two ways: one is through its periodicity, and the other is the detection of its inverse matrix to the inverse transformation [14].

$\boldsymbol{J}_{(X, Y)}^{n+1}=\boldsymbol{A}^{-\mathbf{1}} \underset{\left(X^{\prime}, Y^{\prime}\right)}{n}(\bmod n)$ Equation (3)

Once the encryption algorithm is known, the original image can easily be obtained by iterative computations. So periodicity makes this encryption technique unsecure and Arnold transfer is only suitable for $\mathrm{N} \times \mathrm{N}$ digital images, limits its application.

\subsection{Proposed Security Solution}

To improve the security and extend the application apply random strategy i.e. dividing the image matrix in several overlapping square blocks with random encryption order as shown in the figure 6 . 


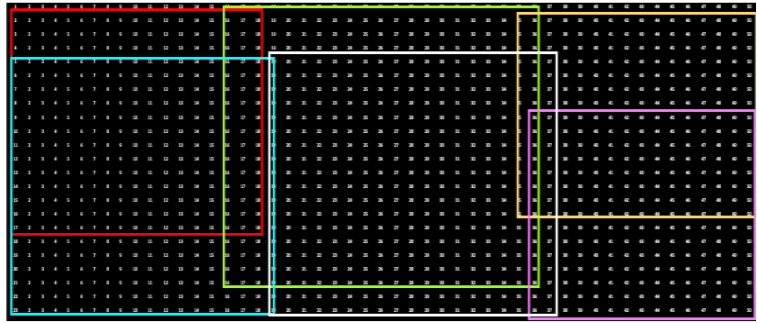

Figure 6: Division of an image

This image encryption scheme with the random Arnold transform is more secure. Since many possibilities of random encryption order, it is difficult for attackers to guess these random orders correctly. Although using Arnold transfer, division of image matrix in blocks covers all pixels by using a series of squares, so there is no size limitation. In addition using Arnold transform for encryption this scheme inherits the robustness of Arnold transform.

\subsection{Objective}

The objective is to test the quality measures: Perceptual Transparency and Robustness of the watermarking technique.

\subsubsection{Objective function 1}

Perceptual transparency means by the presence of watermark the perceived quality of the image should not be degraded. Following visual quality matrix is used for the sake of comparison of the degradation of the watermarked image.

MSE as signal fidelity measure:

$$
\operatorname{MSE}(X, Y)=\frac{1}{N} \sum_{i=0}^{N}(X i-Y i)^{2} \text { Equation (4) }
$$

In the literature of image processing, Mean Square Error is often converted to a peak signal-to-noise ratio (PSNR) measure [15].

$$
P S N R=10 \log _{10} \frac{255^{2}}{M S E} \text { Equation (5) }
$$

Structured Similarity Index (SSIM) is used to measure the similarity between two images. SSIM computes three similarity functions: luminance similarity, contrast similarity, and structural similarity $\mathrm{X}$.

$S(X, Y)=\left(\frac{2 \mu_{X} \mu_{Y}+C_{1}}{\mu_{X}^{2}+\mu_{Y}^{2}+C_{1}}\right) \cdot\left(\frac{2 \sigma_{X} \sigma_{Y}+C_{2}}{\sigma_{X}^{2}+\sigma_{Y}^{2}+C_{2}}\right) \cdot\left(\frac{\sigma_{X Y}+C_{3}}{\sigma_{X} \sigma_{Y}+C_{3}}\right)$ Equation (6)

The higher PSNR and SSIM denote better perceptual quality of watermarked image.

\subsubsection{Objective function 2}

The measure of resistance of watermark against intentional and unintentional attacks such as addition of noise, filtering noise, scaling, translation, resizing, cropping, rotation, etc. is known as Robustness.

\section{Normalized Correlation (NC):}

Normalized correlation is used to measure the similarity and differences between the embedded and recovered watermark

$N C(X, Y)=\frac{\sum_{N=0}^{N-1} \sum_{M=0}^{M-1} X[N, M] . Y[N, M]}{\sqrt{\sum_{N=0}^{N-1} \sum_{M=0}^{M-1} X^{2}[N, M] \sum_{N=0}^{N-1} \sum_{M=0}^{M-1} Y^{2}[N, M]}}$ Equation (7)

Optimization is to maximize the imperceptibility and the robustness i.e. to achieve highest balanced values for PSNR and $\mathrm{NC}$

\section{PROPOSED TECHNIQUE}

The proposed method is implemented in DWT domain. The $3-$ level DWT is applied to the gray scale cover image and the Arnold transform with random strategy is applied on watermark image. The Pseudo random number sequence using key at given independent state is generated. The best wavelet sub-band HLHHHH and HHHHLH are modified to spread the watermark as the PN sequences in the cover image. After the watermark is embedded it is transmitted in the channel.

\subsection{Implementation of Encryption Scheme Using Arnold Transform With Random Strategies}

Step 1: Perform blocking of the image by dividing a whole image matrix in 6 square overlapping blocks such that the union of these 6 blocks should cover all the pixels.

So use three arrays L[6], T[6] and S[6], where

(L[i], T[i]) denote the left-top coordinates of the ith square (i $=1,2, \ldots, \mathrm{n})$

$\mathrm{S}[\mathrm{n}]$ denotes the ith square's size, and $\mathrm{n}$ indicates number of square.

Step 2: Random encryption order:

To improve the security Apply Arnold transform randomly (by 6 pseudorandom with an independent key state) on each of the square block. To produce an ordered sequence, store these $\mathrm{n}$ random numbers in an array $\mathrm{O}$.

Step 3: Encryption:

Apply Arnold transform using equation (2) to O[i]th block.

Then replace this image's $\mathrm{O}[\mathrm{i}]^{\text {th }}$ block with this scrambled block, next apply Arnold transform on $\mathrm{O}[\mathrm{i}+1]^{\text {th }}$ block and again replace the image block with second scrambled block, repeat this steps for all six blocks to generate the scrambled watermark image.

Step 4: Implementation of decryption scheme:

Apply Inverse Arnold transform using equation (3) to $\mathrm{O}[\mathrm{i}]^{\text {th }}$ block where $\mathrm{i}=6$.

Then replace those scrambled pixels in $\mathrm{O}[\mathrm{i}]^{\text {th }}$ block with recovered pixels. Repeat this step for 6 times with $i=n-1$.

\subsection{Watermark Embedding Process}

Input: Cover Image, Watermark message, Gain Factor K Output: Watermarked Image, PSNR, SSIM

Step 1: Read the gray scale Cover Image and determine the size of the Cover Image.

$$
[\mathrm{Mc}, \mathrm{Nc}]=\text { size (cover_object); }
$$

Step 2: using "Haar" wavelet apply 2D-DWT to Cover Object to get the four non overlapping sub bands.

\section{[LL1, LH1, HL1, HH1]=dwt2 (cover_object,'haar');}

Step 3: Apply 2D- DWT to HH and HL sub-band and determine the size of $\mathrm{HH}$ and HL bands

$[\mathrm{MHH} 1, \mathrm{NHH} 1]=\operatorname{size}(\mathrm{HH} 1)$;

$[\mathrm{MHL} 1, \mathrm{NHL} 1]=\operatorname{size}(\mathrm{HL} 1)$;

[HH1LL2,HH1LH2,HH1HL2,HH1HH2]=dwt2(HH1,'haar');

[HL1LL2,HL1LH2,HL1HL2,HL1HH2]=dwt2(HL1,'haar');

Step 4: Apply 2D-DWT to HH1HH2 and HL1HH2 subband and determine the size of HH1HH2 and HL1HH2 subbands

[MHH1HH2,NHH1HH2] = size $(\mathrm{HH} 1 \mathrm{HH} 2)$; 
[MHL1HH2,NHL1HH2] = size $($ HL1HH2);

[HH1HH2LL3,HH1HH2LH3,HH1HH2HL3,HH1HH2HH3]= dwt2(HH1HH2,'haar');

[HL1HH2LL3,HL1HH2LH3,HL1HH2HL3,HL1HH2HH3]=d wt2(HL1HH3,'haar');

Step 3: Set the Gain Factor to $\mathrm{K}$ for embedding.

Step 4: Read in the watermark message, apply Arnold transform with random strategies on watermark message and reshape it to a vector (Message_vector).

Step 5: Generate a CDMA PN sequence using an independent state. (Key image as state of the PN sequence)

Step 6: If the watermark bit is black i.e. zero, add the PN sequence to HH1HH2LH3 and HL1HH2HH3 sub-bands.

Step 7: Perform one level Inverse DWT with new HH1HH2LH3 and new _ HL1HH2HH3, to generate watermarked image:

New_HH1HH2=idwt2(HH1HH2LL3,new_HH1HH2LH3,HH 1HH2HL3,HH1HH2HH3,'haar',[MHH1HH2,NHH1HH2]);

New_HL1HH2=idwt2(HL1HH2LL3,HL1HH2LH3,HL1HH2 HL3,new_HL1HH2HH3,'haar',[MHL1HH2,NHL1HH2]);

Step 8: Perform two level Inverse DWT with new HH1HH2 and new_ HL1HH2, to generate watermarked image:

New_HH1=idwt2(HH1LL2,HH1LH2,HH1HL2, new_HH1HH 2,'haar',[MHH1,NHH1]);

New_HL1=idwt2(HL1LL2,HL1LH2,HL1HL2,new_HL1HH2 ,'haar',[MHL1,NHL1]);

Step 9: Perform one level Inverse DWT with new_ HH1 and new_HL1, to generate watermarked image:

watermarked_img=idwt2(LL1,LH1,new_HL1,new_HH1,'haar ',[Mc,Nc]);

Step 10: Display Watermarked image and PSNR.

\subsection{Watermark Extracting Process}

Input: Watermarked Message, key image

Output: Recovered Watermark, NC

Step 1: Read the watermarked image, and determine the size of the watermarked image.

Step 2: Read the Original Watermark Image, and determine the size of watermark.

Step 3: using "Haar" wavelet apply one level DWT to Watermarked image to separate 'Recovered HL' and 'Recovered HH' band as

[LL, LH, Recovered_HL, Recovered_HH $]$ = dwt2(Watermarked_image,'Haar');

Step4: Apply 2D- DWT to Recovered_HH and Recovered_HL sub-band

[Recovered_HHLL, Recovered_HHLH, Recovered_HHHL, Recovered_HHHH] $=$ dwt2 $($ Recovered_HH,'haar');

[Recovered_HLLL, Recovered_HLLH, Recovered_HLHL, Recovered_HLHH] $=$ dwt2(Recovered_HL,'haar');

Step 5: Apply 2D-DWT to Recovered_HHHH and Recovered_HLHH sub-band and determine the size of Recovered_HHHH and Recovered_HLHH sub-bands
[MRecovered_HHHH, size(Recovered_HHHH);

[MRecovered_HLHH, size(Recovered_HLHH);

[Recovered_HHHHL, Recovered_HHHHHL, dwt2(Recovered_HHHH,'haar');

[Recovered_HLHHLL, Recovered_HLHHHL, dwt2(Recovered_HLHH,'haar')

NRecovered_HHHH] =

NRecovered_HLHH] =

Recovered_HHHHLH, Recovered HHHHHH] =

Step 6: Initialize the Message_vector to all ones.

Step 7: Generate a CDMA PN sequence using an independent state. (Key image as state of the PN sequence). And compute the average correlation coefficient between recovered detail components (Recovered_HHHHLH, Recovered_HLHHHH) and PN sequences.

Step 8: Find the mean correlation and compare it with correlation

for (i=1:length(message_vector))

if $($ correlation(i) $>$ mean (correlation))

message_vector(i) $=0$;

end

end

Step 9: Reshape the message_vector and display the recovered watermark.

\section{EXPERIMENTATION AND RESULTS}

The proposed method is implemented and experiments are carried out using MATLAB. The distinct types of tests are carried out for evaluating the perceptual quality and robustness for gray scale images of $640 \times 426$ size and watermark of 23 X 50 size. Figure 7 shows sample output of 3-level DWT domain based method using Arnold transform with random strategy with original cover image, Watermarked Image in 3-level DWT, 2-level DWT, 1-level DWT, original watermark, extracted watermark and scrambled watermark.

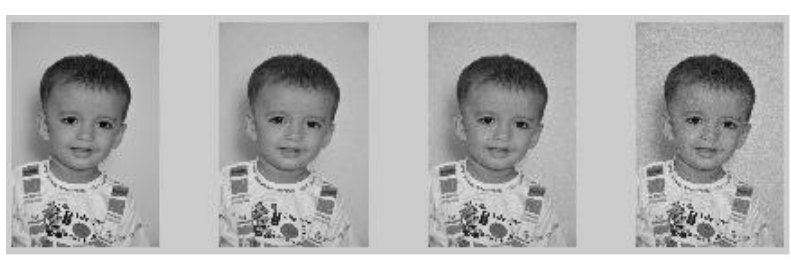

1.

2.

3.

4.

(A)

\section{SHVETI}

(B)
(C)
Figure 7: Sample output of DWT domain based method. (A) 1. Original Cover Image, 2. Watermarked Image in 3level DWT, 3. Watermarked Image in 2-level DWT, 4. Watermarked Image in 1-level DWT. (B) Original Watermark \& Extracted Watermark, (C ) Scrambled watermark 
The performance quality metrics i.e. imperceptibility and robustness is evaluated using equation $3,4,5$ and 6 . Optimization is to maximize the robustness and imperceptibility by high balanced values of NC and PSNR respectively. In this algorithm during embedding process there is only minimal degradation to cover image compared to 2level DWT and 1-level DWT and easily the small watermark is retrieved from the watermarked image.

\subsection{Comparative Performance Analysis}

Using the algorithmic steps proposed in the method (Munesh Chandra et.al. 2010) [4] is run in our experimental setup. The observations are shown in the following figures. As shown in the figure8 the proposed technique supports high perceptual quality compared to method (Munesh Chandra et.al. 2010) [4], 1-level DWT \& 2-level DWT i.e. the better quality of the watermarked image is maintained through proposed method.

\subsection{Robustness Test}

The proposed technique has also achieved high robustness under variety of attacks. The out coming results including extracted watermarks, PSNR, NC are presented in Table1.

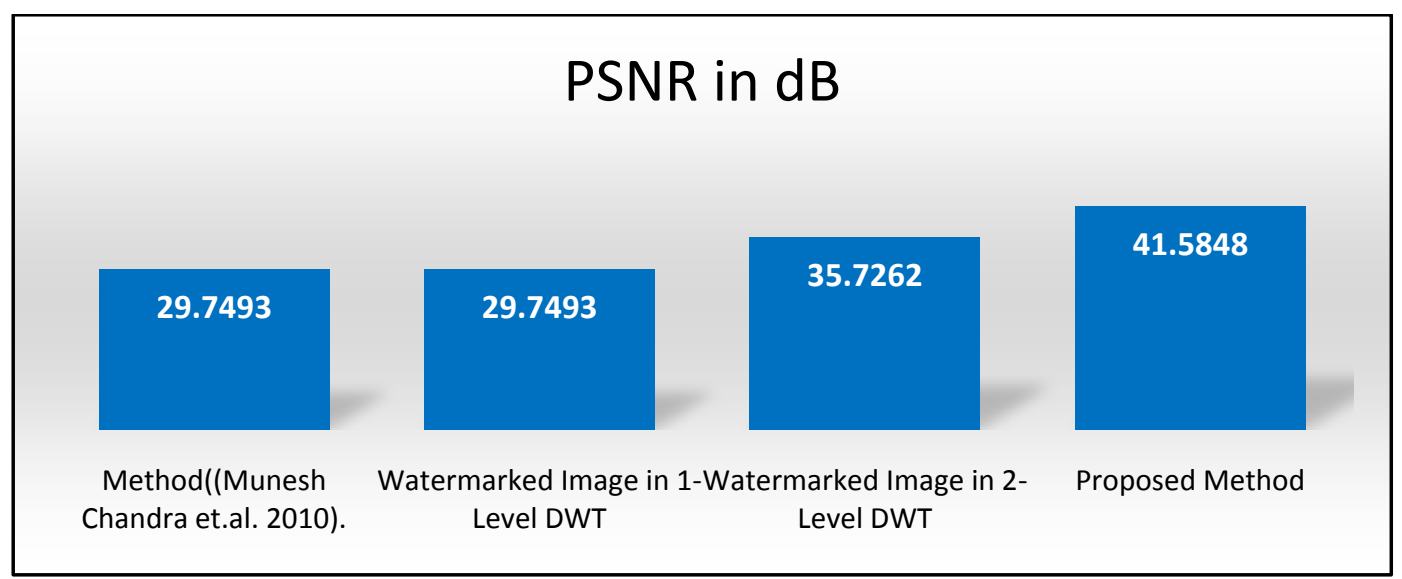

Figure 8: Comparative of imperceptibility in terms of PSNR in dBs for proposed method, Method (Munesh Chandra et.al. 2010) [4], Watermarked Image in 1-Level DWT, and Watermarked Image in 2-Level DWT

Table 1: Extracted watermark, PSNR for watermarked_image, SSIM for Watermarked image, NC for extracted_watermark under several noise attacks and noise filters.

\begin{tabular}{|c|c|c|c|c|}
\hline Attack type & $\begin{array}{c}\text { Extracted } \\
\text { Watermark }\end{array}$ & $\operatorname{PSNR}(\mathrm{dB})$ & SSIM & NC \\
\hline No Attack & Asis & 54.193 & 0.953 & 0.663 \\
\hline $\begin{array}{c}\text { Salt \& Pepper } \\
(\text { Density }=0.001)\end{array}$ & Wing & 54.1031 & 0.9241 & 0.6575 \\
\hline $\begin{array}{c}\text { Salt \& Pepper } \\
\text { (Density=0.01) }\end{array}$ & 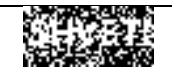 & 53.5735 & 0.8186 & 0.6179 \\
\hline Rotation by 0.1 & 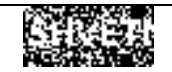 & 53.8163 & 0.9627 & 0.6336 \\
\hline Resize Scale by 0.999 & Not & 54.0151 & 0.9402 & 0.6521 \\
\hline Resize Scale by 2 & 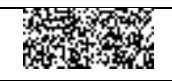 & 51.0266 & - & 0.3061 \\
\hline Cropping 1 X 1 & A & 54.1779 & - & 0.6623 \\
\hline Cropping 1 X 5 & Pres & 54.1478 & - & 0.661 \\
\hline median filter $2 \times 2$ & 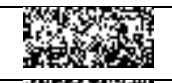 & 52.6865 & 0.9294 & 0.5361 \\
\hline median filter $3 \times 3$ & We & 52.367 & 0.9451 & 0.5016 \\
\hline $\begin{array}{c}\text { Gaussian } \mathrm{M}=0 \text { \& } \\
\mathrm{V}=0.001\end{array}$ & ity & 54.0296 & 0.61 & 0.6494 \\
\hline $\begin{array}{c}\text { Gaussian } \mathrm{M}=0 \text { \& } \\
\mathrm{V}=0.005\end{array}$ & 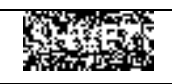 & 53.4062 & 0.3094 & 0.5915 \\
\hline
\end{tabular}


The method shows significance achievement of robustness in 3-Level DWT domain (HLHHHH \& HHHHLH sub-band) against several possible noise addition like salt \& Pepper noise with density 0.01 , salt \& Pepper noise with density 0.001 , Rotation by 0.1 attack, Resize scale by 0.999 attack,
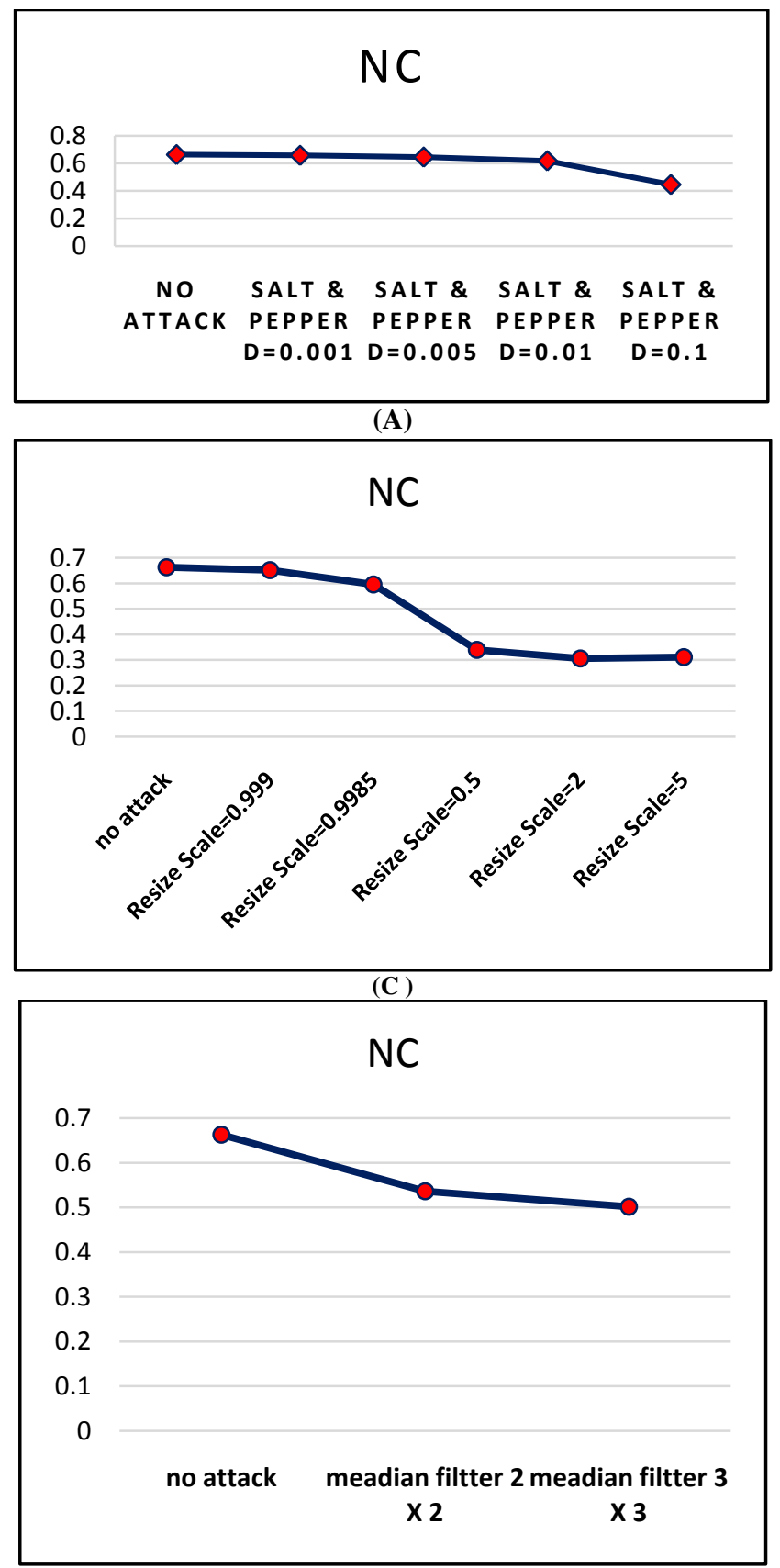

(E) resize scale by 2 attack, Cropping 1 x 1 attack. Cropping 1 x 5 attack, filtering attacks like Median filtering $2 \times 2$, Median filtering $3 \times 3$, Gaussian filter with $\mathrm{M}=0$ \& $\mathrm{V}=0.001$, Gaussian filter with $\mathrm{M}=0$ \& $\mathrm{V}=0.005$ shown in the figure9.

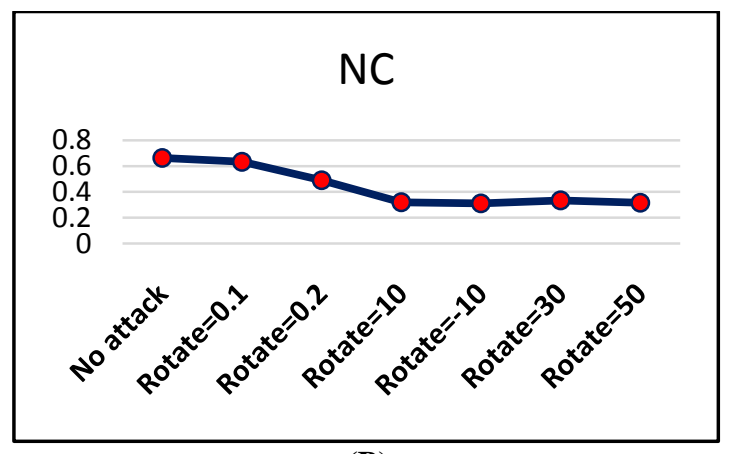

(B)

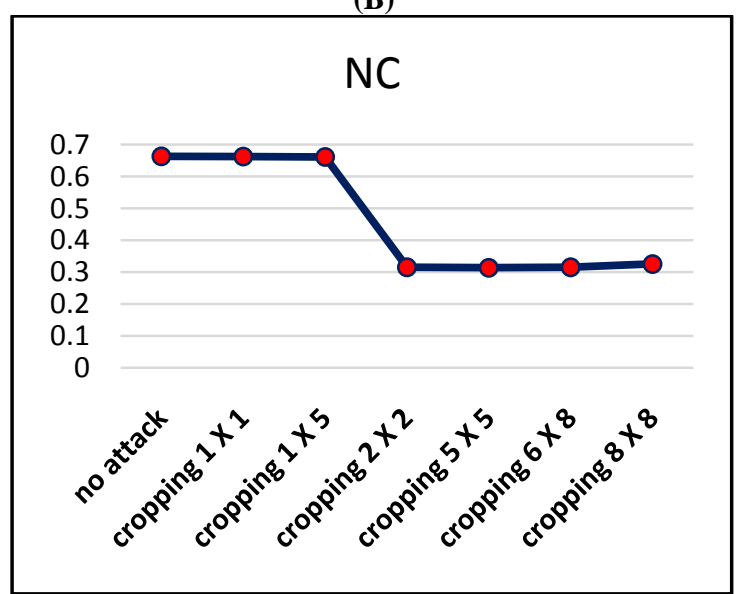

(D)

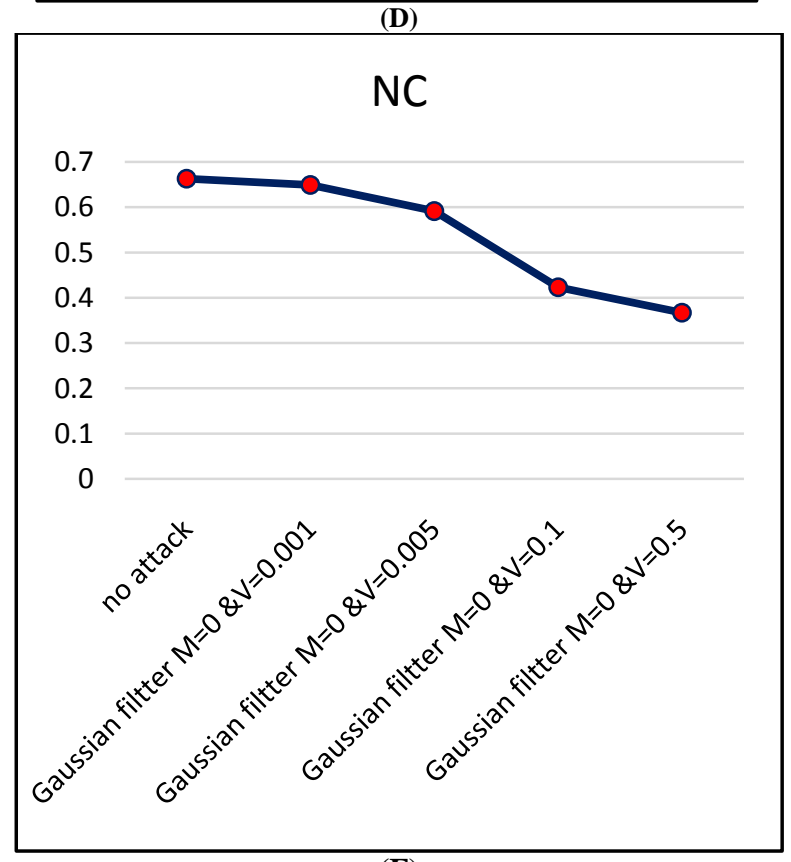

(F)

Figure 9: Robustness against Salt \& Pepper Noise (A), Rotation (B), Cropping (C), Resizing (D), Median Filtering (E), Gaussian Filter Noise (F)

The Proposed method is also found more robust compared to Method (Munesh Chandra et.al. 2010) [4], for various noise addition and filtering attacks, as comparatively in method (Munesh Chandra et.al. 2010) [4], the NC value at different noise attack differs more with $\mathrm{NC}$ value at no attack compared to the proposed method. This is shown in the figure 10 by comparing the $\mathrm{NC}$ value at different noise attack with the NC value at no attack. 


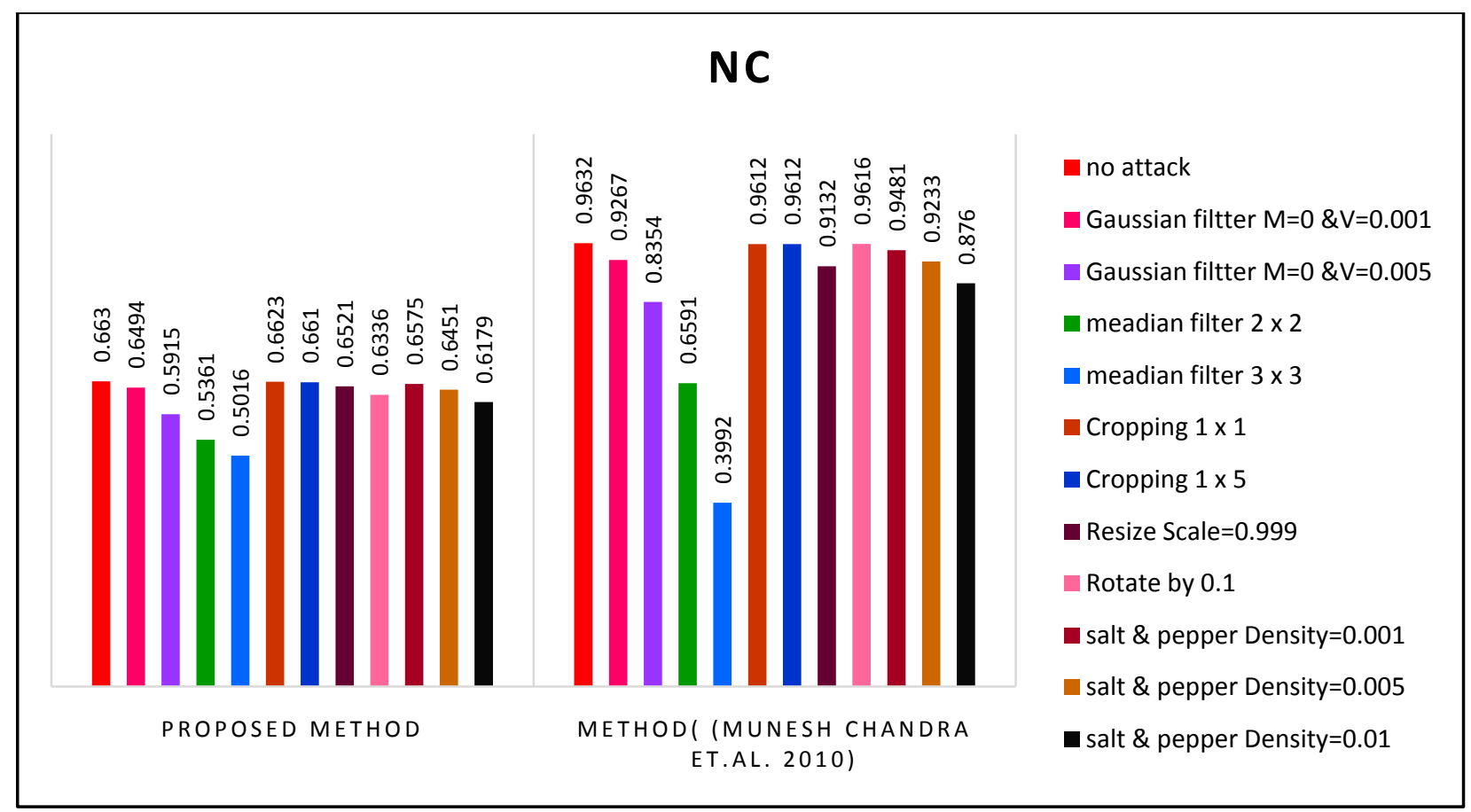

Figure 10: Comparing robustness of proposed method with Method (Munesh Chandra et.al. 2010) [4], for various noise addition and filtering attacks

The experimental results evidently show that proposed technique i.e. 3-level DWT with best sub-band HLHHHH and
HHHHLH is faster with compared to 2-level DWT, 1-level DWT and Method (Munesh Chandra et.al. 2010) [4].

Table 2: Comparison of computation time i.e. embedding time plus extraction time in unit seconds)

\begin{tabular}{|c|c|c|c|}
\hline Proposed Method & 2-Level DWT & 1-Level DWT & $\begin{array}{c}\text { Method (Munesh Chandra } \\
\text { et.al. 2010) [4] }\end{array}$ \\
\hline $\mathbf{3 . 0 1 0 8}$ & 9.9528 & 40.9191 & 41.3871 \\
\hline
\end{tabular}

Based on the experiment result the observations can be summarized as:

The proposed technique supports high perceptual quality compared to method (Munesh Chandra et.al. 2010) [4], 1level DWT \& 2-level DWT.

The Proposed method is also found more robust compared to Method (Munesh Chandra et.al. 2010) [4], for various noise addition and filtering attacks.

The proposed technique is faster with compared to 2-level DWT, 1-level DWT and Method (Munesh Chandra et.al. 2010) [4].

\section{CONCLUSION}

The challenging issues of Digital watermarking are to achieve imperceptibility, robustness and security. This proposed method fulfills these requirements. The performance of the proposed method is found better than the referenced method using LH \& HL sub-bands. The proposed method is robust to various attacks and noise addition. This is more secure than other existing methods. This method can be extended for medical images, color images and video watermarking. The crucial part of biomedical images is the image quality. The current proposed system can further be extended to provide more imperceptible, robust and secured system for medical image watermarking.

\section{REFERENCES}

[1] Matthieu Urvoy. Dalila Goudia, and Florent Autrusseau."Jul. 2014. Perceptual DFT Watermarking
With Improved Detection and Robustness to Geometrical Distortions", IEEE Transactions on Information Forensics and Security, Vol. 9, No. 7, pp. 1108-1119.

[2] Ramani K.; Prasad E.V, Varadarajan S.; Subramanyam A,Dec. 2008."A Robust Watermarking Scheme for Information Hiding", Advanced Computing and Communications, 16th International Conference, 14-17 pp: $58-64$.

[3] Raval Mehul, Rege Priti, 2003. "Discrete wavelet transform based multiple watermarking schemes", Conference on convergent technologies for Asia-pacific region, vol. 3, pp. 935-938

[4] Munesh Chandra, Shikha Pandey, 2010. "A DWT Domain Visible Watermarking Techniques for Digital Images", 2010 International Conference on Electronics and Information Engineering.

[5] Nikita Kashyap, G. R. SINHA, April 2012."Image Watermarking Using 3-Level Discrete Wavelet Transform (DWT)", in I.J.Modern Education and Computer Science, 2012, 3, 50-56.

[6] C. Pradhan, V. Saxena, A. Bisoi, October 2012. "Imperceptible Watermarking Technique using Arnold's Transform and Cross Chaos Map in DCT Domain", International Journal of Computer Applications (0975 8887) Volume 55- No.15.

[7] AI-Asmari, A., Salama, A., Iliyasu, A., Al-Qodah, M. 2012. A DWT ordering scheme for hiding data in images 
using pixel value difference. In: IEEE Eighth Int. Conf. on Computational Intelligence and Security (CIS), pp. $553-557$

[8] A. Nag, J.P. Singh, S. Khan, S. Biswas, and D. Sarkar, 2011. "Image Encryption Using Affine Transform and XOR Operation", in Proc. international conference on Signal Processing, Communication, Computing and Networking Technologies (ICSCCN), pp.309-312.

[9] Zhang Yanqun; Wang Qianping; 2009. "A New Scrambling Method Based on Arnold and Fermat Number Transformation" International conference on Environmental science and information application technology.

[10] M. Mohammed Sathik and Sujatha S.S, 2010. "An Improved Invisible Watermarking Technique for Image Authentication", International Journal of Advanced Science and Technology, Vol-24, Pp-61- 74

[11] A. Ravindran, A. Jayaram, Ambily N, B.M. John, October 2014."Hiding Information in Medical X-ray
Images", International Journal of Advanced Information *Science and Technology (IJAIST), Vol.30, No.30.

[12] Poulami Ghosh, Rilok Ghosh, Souptik Sinha, Aruna Chakroborty 2012. "A Novel Digital Watermarking Technique for Video Copyright Protection" CS \& IT 06, pp. 601-609.

[13] S. Hore, T. Bhattacharya, S. R. Bhadra Chaudhuri December 2013. "A Robust Medical Image Authentication Technique using QR Code and DWT" International Journal of Computer Applications (0975 8887) Volume 83 - No.16.

[14] Min Li, Ting Liang, Yu-jie He 2013. "Arnold Transform Based Image Scrambling Method" 3rd International Conference on Multimedia Technology.

[15] Zhou Wang and Alan C. Bovik JANUARY 2009 "Mean Squared Error: Love It or Leave It? [A new look at signal fidelity measure]" IEEE SIGNAL PROCESSING MAGAZINE vol. 26, no. 1, pp. 98-117. 